\title{
A risk-stratified approach toward safely resuming OnabotulinumtoxinA injections based on dosing and ambulatory status in pediatric patients with cerebral palsy during the Coronavirus pandemic of 2019 (COVID-19)
}

\author{
Matthew J. McLaughlin ${ }^{\mathrm{a}, *}$, Mark T. Fisher ${ }^{\mathrm{a}}$, Sathya Vadivelu ${ }^{\mathrm{a}}$, Justin Ramsey ${ }^{\mathrm{a}}$, Denesh Ratnasingam ${ }^{\mathrm{b}}$ \\ Emily McGhee ${ }^{\mathrm{c}}$ and Kim Hartman ${ }^{\mathrm{a}}$ \\ ${ }^{a}$ Children's Mercy Hospital Kansas City, MO, USA \\ ${ }^{\mathrm{b}}$ Rehabilitation Institute of Michigan, Detroit Medical Center, Wayne State University, Detroit, MI, USA \\ ${ }^{\mathrm{c}}$ University of Missouri, Columbia, MO, USA
}

\begin{abstract}
.
PURPOSE: After the onset of the Coronavirus pandemic of 2019-2020 (COVID-19), physicians who inject OnabotulinumtoxinA (BoNT-A) were left with determining risks and benefits in pediatric patients with cerebral palsy. Many of these patients have pre-existing conditions that make them more prone to COVID-19 symptoms, and this susceptibility potentially increases after BoNT-A injections.

METHODS: A retrospective chart review of 500 patients identified 256 pediatric patients with cerebral palsy who received an intramuscular BoNT-A injection to determine relative doses used for each Gross Motor Functional Classification Score (GMFCS). Data regarding age, weight, GMFCS, BoNT-A total body dosage, and inpatient hospitalizations for 6 months post-injection were collected. Differences between GMFCS levels were analyzed using one-way analysis of variance testing. Inpatient hospitalizations were recorded and assessed using relative risk to determine the population risk of hospitalization in the setting of initiating injections during the COVID-19 pandemic.

RESULTS: Based on GMFCS level, patients who were GMFCS I or II received fewer units of BoNT-A medication per kilogram of body weight compared to GMFCS III-V ( $p<0.0005, F=25.38)$. There was no statistically significant difference in frequency or time to hospitalization when comparing patients receiving BoNT-A compared to a control group.

CONCLUSIONS: Resumption of BoNT-A injections during the time of COVID-19 requires a systematic approach based on risks and potential benefits. Data from this analysis does not show increased risk for patients who received injections historically; however, recommendations for resumption of injections has not previously been proposed in the setting of a pandemic. In this manuscript, a tiered approach to considerations for injections was proposed. Botulinum toxin type A injections have a history of improving spasticity in the pediatric patient with cerebral palsy. Ensuring appropriate selection of patients for injection with BoNT-A during this pandemic is increasingly important.
\end{abstract}

Keywords: Cerebral palsy, botulinum toxins, drug-related side effects and adverse reactions, adverse events, COVID-19

* Corresponding author: Matthew J. McLaughlin, Children's Mercy Hospital, 2401 Gillham Road, Kansas City, MO, 64108, USA. Tel.:
+1 816234 1570; Fax: +1 16983 9845; E-mail: mjmclaughlin@ cmh.edu. 


\section{Introduction}

The novel coronavirus disease 2019 (COVID-19) pandemic has forced many healthcare facilities to reconsider typical operating procedures to ensure the safety of patients, families, staff, and the public. In March 2020, the American College of Surgeons (ACS) and American Academy of Physical Medicine and Rehabilitation (AAPM\&R) recommended that all scheduled elective procedures be minimized, postponed, or canceled until further assessment by the Center for Disease Control and Prevention (CDC) [1,2]. Botulinum toxin injection procedures, including type A formulations (Botox ${ }^{\circledR}$, Dysport ${ }^{\circledR}$, Xeomin $^{\circledR}$ ) and type B formulations (Xeomin ${ }^{\circledR}$ ), in children with cerebral palsy are mainly considered non-urgent procedures and have been placed on hold at many institutions. As healthcare systems and public places are preparing to reopen at full capacity, there are no clear guidelines on resumption of Botulinum toxin type A procedures in children. This paper discusses the experience at a tertiary care children's hospital and extrapolates from these findings the risks to consider when performing Botulinum toxin type A injections during a pandemic and a phased approach to restarting Botulinum toxin type A procedures based on available data.

Due to differences in testing and reporting patterns, the exact number of children infected with the virus is unknown, with best estimates of 1.7-3.6\% of positive cases in the United States [3]. Most children appear to have milder symptoms, including fever, cough, and shortness of breath, while many are asymptomatic [4]. Among hospitalized and critically ill children with COVID-19, 77-83\% had preexisting comorbidities [4,5]. At baseline, children with cerebral palsy have higher rates of hospital admission than peers [6]; thus they may be at even higher risk of hospitalization or more severe illness in the setting of COVID- 19 .

Botulinum toxin type A produces local muscle weakness as a treatment for hypertonicity in children with cerebral palsy. For this patient population, severe adverse events with the use of Botulinum toxin type A can include respiratory issues, dysphagia, aspiration, generalized weakness, and even death - especially in patients with a history of dysphagia, gastrostomy, and/or history of aspiration pneumonia [7-10]. Such adverse events can result in unplanned hospitalization and appear to be associated with GMFCS level and Botulinum toxin type A dose [7-10]. In the setting of a predominantly respiratory viral illness such as COVID-19, these factors are important to consider when resuming Botulinum toxin type A injections.
Because variation exists in practice patterns related to OnabotulinumtoxinA (BoNT-A), we present a review of practices at a single institution related to dosing, gross motor functional classification system (GMFCS) level, and inpatient hospitalizations (as a potential adverse event related to BoNT-A). We hypothesized that patients with a higher GMFCS score (less ambulatory) would receive a larger dose of BoNT-A compared to those who had a lower score (more ambulatory), and, thus, these patients would be a higher risk for hospitalization and at greater risk for contracting COVID-19. The primary goal of this manuscript was to propose a tiered approach for resuming BoNT-A procedures utilizing available data at this center during the COVID-19 pandemic.

\section{Methods}

After institutional board review approval was obtained, pediatric aged patients with cerebral palsy were identified using an informatics search for a computerized order entry, which was previously part of a departmental quality improvement project by physicians to identify patients by GMFCS. Inclusion criteria for this project were patients who had entry of this GMFCS order documented from March 1, 2013 until September 30, 2014. This time period represented the highest frequency of use of this computerized order. Patients needed to be between ages 1 through 21 during the time of this order. Further inclusion in this review included patients seen by a rehabilitation medicine provider or neurologist who performed intramuscular BoNT-A injections. Five hundred patients were identified for inclusion in this study; however, 492 patients met the diagnostic inclusion criteria for analysis. Two hundred fifty six of the 492 patients had received injections with BoNT-A and were included in the analysis. Data regarding age, weight, GMFCS, total BoNT-A dosage, and any data regarding an inpatient admission within 6 months after injections were performed. At the institution in this study, only OnabotulinumtoxinA (BoNTA) was primarily used for treatment of spasticity during the 2013-2014 time period. Patients who did not receive BoNT-A injections were analyzed for any inpatient hospitalization within 6 months of seeing a rehabilitation medicine provider. Reasons for not meeting inclusion criteria included inadequate documentation to undergo analysis or change in diagnosis (i.e. new diagnosis of chromosomal abnormality). Statistical analysis for dosage comparison between GMFCS levels was 
Table 1

Dosing of botulinum toxin type-A per kilogram for each GMFCS group

\begin{tabular}{ccccc}
\hline GMFCS group & Number of patients & Mean dose of BoNT-A injected per kg & Standard deviation & Maximum injected dose per kg \\
\hline 1 & 36 & 5.02 & 2.99 & 12.5 \\
2 & 63 & 5.33 & 2.60 & 12.0 \\
3 & 48 & 8.97 & 3.97 & 20.13 \\
4 & 43 & 11.02 & 5.23 & 24.84 \\
5 & 65 & 11.15 & 4.99 & 24.10 \\
\hline
\end{tabular}

performed using a one-way ANOVA. Subsequent between group comparisons were performed using multiple comparison testing. Kaplan-Meier analysis was performed to plot any differences in time to admission.

Inpatient admissions data were collected and included if they occurred within 6 months of either receiving injections of intramuscular BoNT-A or having a visit with rehabilitation medicine for all participants in this study. Length of time since having injections was also documented. Relative risk ratios were calculated to determine any differences in rate of admission within seeing a rehabilitation provider. Statistical calculations were performed with $\mathrm{IBM}^{\circledR}$ SPSS $^{\circledR} 23$.

\section{Results}

The doses of BoNT-A injected in this patient population varied from GMFCS I patients receiving the lowest total body dose (mean 5.02, standard deviation \pm 2.99 units $/ \mathrm{kg}$ ) increasing up to GMFCS V patients who received the highest total body dose (mean 11.16 \pm 4.99 ). Table 1 shows the number of patients who received Botulinum toxin injections, the average total body dosage and the standard deviation for each GMFCS group. There was a statistically significant difference $(F=26.71, p<0.0005)$ between group comparisons. Multiple comparison testing indicated a statistically significant difference $(p<0.0005)$ between GMFCS groups I-II compared to groups III-V. Groups III-V showed no statistically significant differences between each other $(p>0.05)$ when multiple comparisons testing was performed. This comparison is highlighted in Fig. 1. The maximum dose of BoNT-A injected in this group were in a GMFCS IV patient who received 24.84 units $/ \mathrm{kg}$ and a GMFCS V patient who received 24.10 units $/ \mathrm{kg}$.

Of the 256 patients who received BoNT-A during this study, 23 (8.9\%) were hospitalized during the 6 months after BoNT-A injection. This compares with 17 (7.2\%) of the 236 patients with cerebral palsy who did not receive BoNT-A injections. GMFCS V was the most common group requiring hospitalization in both the in-

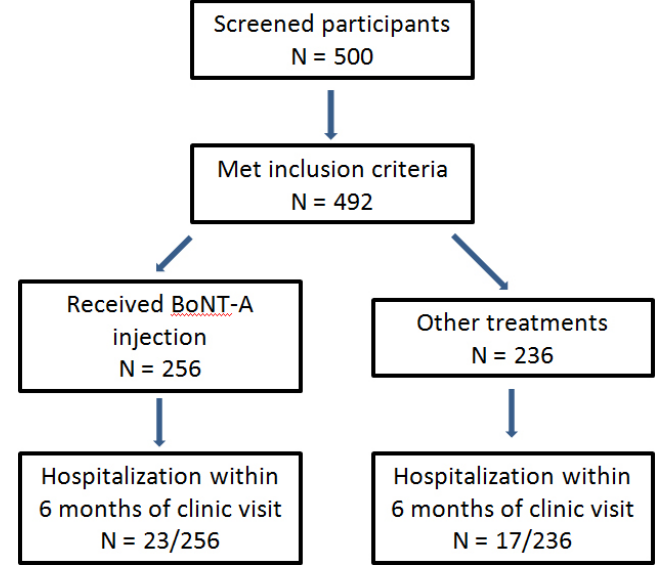

Fig. 1. Demographics of participants in this study.

jected ( $n=17$ ) and non-injected $(n=8)$ group. The relative risk of hospitalization within six months after either receiving BoNT-A injections or being seen by a rehabilitation provider was 1.22 , which was not statistically significant ( $p=0.51)$. Kaplan-Meier curve (Fig. 2) depicts the time to hospitalization after injection in the exposed group versus time after seeing a rehabilitation provider in the comparison, non-exposed group.

\section{Discussion}

In the process of creating a tiered approach toward safely resuming and scheduling patients in need of BoNT-A injections, dose of BoNT-A was initially considered a significant risk factor that was at the forefront of the conversation regarding which patients would be safest to resume BoNT-A injections. This evaluation demonstrates that patients in GMFCS categories with decreasing functional ambulation (GMFCS III-V) received increased doses of BoNT-A in practice compared to those who were more functionally mobile (GMFCS I-II). On average, GMFCS IV and V patients received greater than 10 units per kilogram. Despite patients with GMFCS scores IV-V receiving higher doses of BoNT-A than patients with a GMFCS score of I-III, 


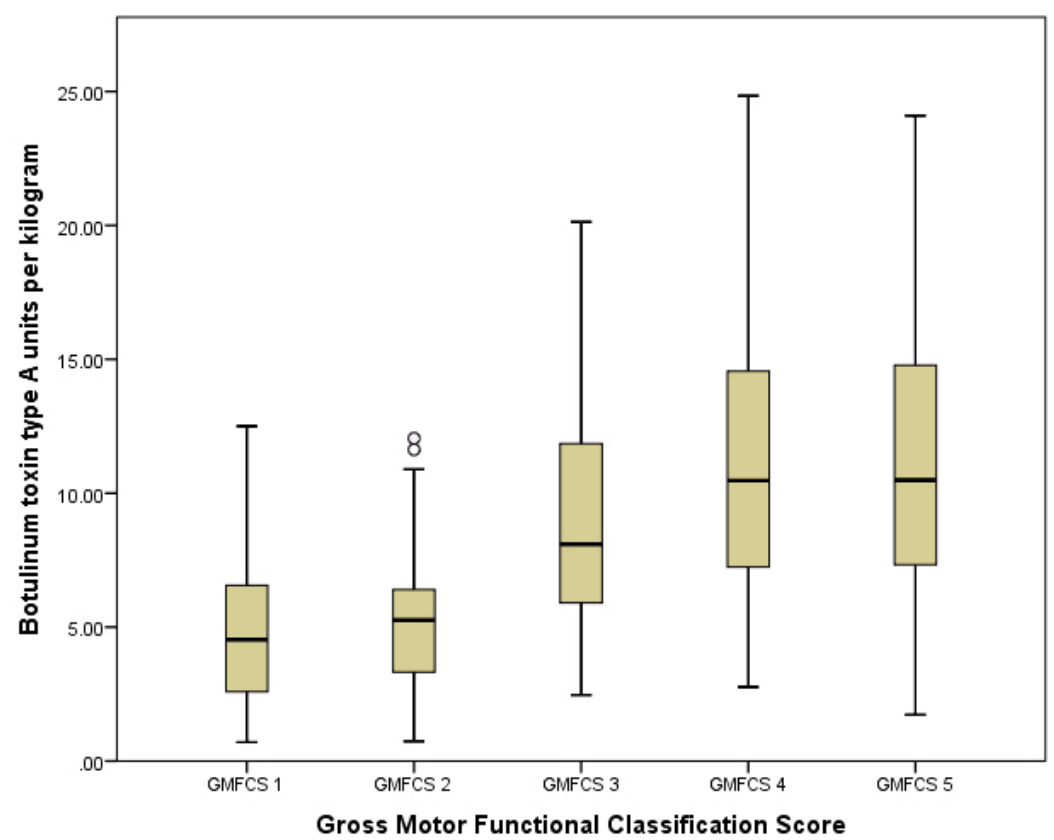

Fig. 2. Comparsion between GMFCS groups. This boxplot shows the total body amount of botulinum toxin type A injected per GMFCS functional group.

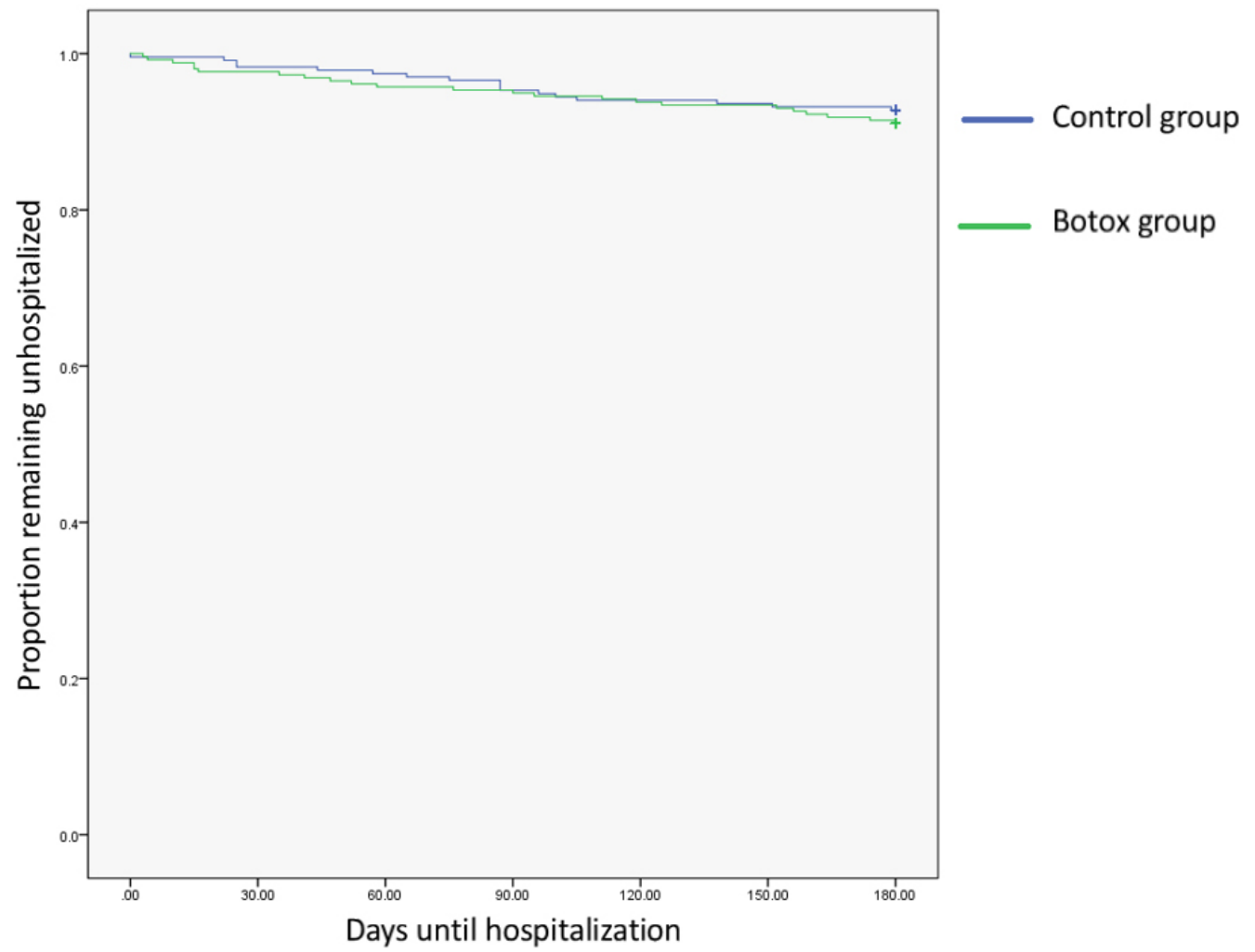

Fig. 3. Kaplan-Meier curve of time to admission after receiving botulinum toxin type-A. Participants in both groups experiences a high rate of hospitalization during the observation period; however, the differences between the two groups was not significant. 
less ambulatory patients did not appear to be more at risk for being hospitalized within 6 months after the injections. This is despite this less ambulatory population being at greater risk for admission to inpatient units. Part of the challenge of evaluating adverse events encountered in cerebral palsy is the high rate of preexisting medical problems in this population, such as dysphagia. Pediatric patients with cerebral palsy are 4.3 times higher likelihood of being hospitalized compared to their age-matched peers $[6,11]$. The results of this study show that patients who are less mobile are exposed to higher doses of BoNT-A at the institution included in this study.

In addition to the potential health risks of COVID-19 in the setting of injecting medically complex patients with BoNT-A, the risks of the procedure itself were considered for both patients and staff when determining the tiered approach to be utilized in this hospital system. The mode of COVID-19 transmission is not completely understood, but it is generally believed to be via droplet, contact, or aerosolized particles from an infected individual speaking, coughing, or sneezing [12]. Particles appear to travel short distances and can infect others via mouth, nose, eye, or possibly inhalation contact [12]. Appropriate personal protective equipment (PPE), which may include a facemask, eye protection, gown, gloves, and/or N95 mask, is necessary to ensure safety of children and healthcare providers. The authors recommend mask wearing for all procedural patients, but also recommend wearing face shields or goggles along with a face mask for providers. Goggles appear to be most needed in situations where non-sedated patients may have increased potential for yelling or crying during injections. Selection of PPE should also be based on national and institution guidelines and depends on risk related to the encounter and procedure.

There should be consideration for likelihood of aerosolization/contact/droplet spread, proximity of healthcare providers, and type of analgesia/sedation impact selection of PPE. There is practice variability regarding how BoNT-A procedures are performed at different institutions. Sedation and pain management during BoNT-A procedures range from general anesthesia, sedation, oral medications, topical medications, or distraction alone [13]. These procedure-related factors also need to be considered when resuming BoNT-A procedures.

By considering general risks to more medically complex patients, dose of BoNT-A injected, number and location of injection sites, and method of localization, a tiered approach toward a strategic resumption of BoNT-
A is proposed (Table 2). These tiers represent a thoughtful and standard approach toward scheduling patients in a manner that would allow risk stratification based on several different factors. Medication risk, patient exposure risk, staff exposure risk, and the general procedural risks were considered when creating these tiers. Level 1 represents a low-risk injection procedure, with a patient who can comply by wearing a mask and following instructions and does not require physical restraint during the injection. Furthermore, this level represents a familiar patient where very few injections would need to be performed; therefore, injecting physicians would likely not be in close contact for a prolonged period of time. As a patient would move to a higher tier, more supportive staff or PPE might be utilized. For example, patients in Tier 3 or 4 would have a higher possibility of passing along COVID as they are either unable to wear a mask or have a higher propensity to produce more droplets during a procedure. This tiered system allows for individual evaluation of risk based on the factors deemed most important to note prior to performing Botulinum toxin injections. Despite COVID related risks associated with performing BoNT-A injections, significant harm may come from not performing these injections. Inappropriate treatment of spasticity during this time could greatly affect function, cause contracture, or led to increased pain in children who would have otherwise received regular injections. This tiered approach represents a thoughtful, yet cautious, method toward safely resuming injections. Although initially proposed for this ongoing pandemic, it would also serve a role for more sophisticated clinical scheduling in the future.

In this study, one limitation was that patients were included for analysis for all cause hospitalizations at a time that was prior to the pandemic, which is challenging in a population that has higher rates of hospitalization compared to healthy controls [6]. All of the patients had received only one formulation of Botulinum toxin (OnabotulinumtoxintypeA) injections in this study, so correlation with recently approved dosing guidelines for other formulations of Botulinum toxin injections would be needed. Additionally, there has been a relatively lower incidence at this institution compared to national reports with 816 patients testing positive out of 19,529 tested patients at the time of this writing. At other institutions with a higher incidence, a more cautious approach may be warranted Improved reporting of adverse effects of botulinum toxin and future prospective studies analyzing risk will be needed to provide better understanding of the risk of all botulinum toxins commonly injected in pediatrics. 
Table 2

Risk can be characterized in many ways but the authors have chosen to define the impact of the pandemic on onabotulinumtoxinA injections in the following ways: Medication Effect Risk: dose, location of injections, systemic spread, respiratory insufficiency, dysphagia, novel and incompletely understood pathologic impact of the virus on physiologic systems. Patient Exposure Risk: factors that place this patient population at greater than average population risk of adverse outcomes from SARS-CoV-2 such as medical co-morbidities, functional impairment, decreased respiratory reserve and airway clearance. Staff Exposure Risk: likelihood of patient resistance requiring manual staff restraint, crying or other forceful exhalation increasing velocity of droplet dispersion, availability of medical grade PPE, ability to accommodate social distancing in work spaces. Procedural Risk: this has a direct modulatory impact on the three other risk factors and is comprised of factors that affect the duration and complexity of the procedure such as sedation, guidance modalities, and number of injections. This also includes the capacity of the facility to screen for signs and symptoms of infection and allow for social distancing at check points and waiting rooms.

\begin{tabular}{|c|c|c|c|c|}
\hline & Patient Exposure Risk & Staff Exposure Risk & Medication Effect Risk & Procedural Risk \\
\hline \multirow[t]{2}{*}{ Level 1} & Low co-morbidities & Patient can wear mask & Total dose $<10$ units $/ \mathrm{kg}$ & Anatomic, EMG, e-stim localization \\
\hline & Patient can wear mask & $\begin{array}{l}\text { No need for close contact to } \\
\text { position }\end{array}$ & & $\begin{array}{l}\text { Familiar patient and family/patient } \\
\text { feel comfortable with injector } \\
0-\leqslant 5 \text { injection sites }\end{array}$ \\
\hline \multirow[t]{2}{*}{ Level 2} & Low co-morbidities & Patient can wear mask & Total dose $10-15$ units $/ \mathrm{kg}$ & $\begin{array}{l}\text { Anatomic, EMG, e-stim or } \\
\text { Ultrasound localization }\end{array}$ \\
\hline & Patient can wear mask & $\begin{array}{l}\text { Low } / \text { moderate chance of } \\
\text { needing close contact for } \\
\text { positioning }\end{array}$ & & $\begin{array}{l}\text { Unfamiliar patient or family/patient } \\
\text { discomfort with a new provider }\end{array}$ \\
\hline \multirow[t]{3}{*}{ Level 3} & $\begin{array}{l}\text { Higher co-morbidities (i.e. history } \\
\text { of aspiration pneumonia, G-tube } \\
\text { feeding requirements, etc.) }\end{array}$ & Patient cannot tolerate mask & Total dose $>15$ units $/ \mathrm{kg}$ & $\begin{array}{l}5-\leqslant 10 \text { injection sites } \\
>10 \text { injection sites }\end{array}$ \\
\hline & Patient cannot tolerate mask & Expected to resist/cry & $\begin{array}{l}\text { Thoracic injections: such as } \\
\text { pectoralis, teres major, } \\
\text { trapezius, latissimus }\end{array}$ & \\
\hline & & May need $2+$ staff to position & & \\
\hline Level 4 & $\begin{array}{l}\text { Decreased respiratory reserve } \\
\text { (such as suctioning, trach, O2 at } \\
\text { baseline) }\end{array}$ & & $\begin{array}{l}\text { Injections for cervical } \\
\text { dystonia/torticollis }\end{array}$ & \\
\hline
\end{tabular}

* Any 2 criteria of level 1-3 requires stratification to the higher risk. Any single criteria in level 4 requires a level 4 stratification. ${ }^{* *}$ Exception: functional, medically urgent, and quality of life issues may be carefully considered at any point after a thorough discussion between provider and guardian.

In addition to a tiered approach in conceptualizing botulinum toxin injection risk, the frequency of injections should also be considered. There is an increasing amount of evidence in recent years that more traditional injection frequencies of three to four months in pediatric populations may not be necessary $[14,15]$. At least two studies indicate that the clinical efficacy of botulinum toxin injection frequencies of 12 months may be as beneficial as every three to four month injections [14-16]. Considering the change in practice that COVID-19 is causing throughout medicine, it is important for providers to carefully assess the frequency of botulinum injections in the context of overall risk and the individual patient's goals for injections.

\section{Conclusion}

With the recent COVID-19 pandemic, specific concerns exist in the pediatric population with cerebral palsy. As Botulinum toxin type A injections have a history of improving spasticity in the pediatric patient with cerebral palsy in the short term, continued evaluations of dosing practices and adverse events allows for safer utilizations of these medications. A tiered approach for utilizing both appropriate dosing and personal protective equipment will help identify appropriate patients to inject.

\section{Acknowledgments}

This project was funded through a T32 fellowship grant in Clinical Pharmacology (NIH T32HD06903806) (McLaughlin) and NIH K12 program (NIH K12HD 093427-01) (McLaughlin). None of the other authors have any relevant financial disclosures.

\section{Conflict of interest}

None of the authors have conflicts of interest other than the federal funding listed above. 


\section{References}

[1] Surgeons. ACo. COVID-19: Recommendations for Management of Elective Surgical Procedures. 13 March 2020. Available from: https://www.facs.org/covid-19/clinical-guidance/ elective-surgery.

[2] Rehabilitation AAoPMa. COVID-19: Statement from the AAPM\&R Board of Governors 20 March 2020. Available from: https://www.aapmr.org/docs/default-source/news-andpublications/academy-statement---covid-19-final.pdf?sfvrsn $=\mathrm{d} 58 \mathrm{a} 5 \mathrm{f} 7 \mathrm{c} \_0$

[3] CDC Coronavirus Disesase 2019: Cases, Data, \& Surveillance [May 26, 2020]. Available from: https://www.cdc.gov/corona virus/2019-ncov/cases-updates/cases-in-us.html.

[4] Team CC-R. Coronavirus Disease 2019 in Children - United States, February 12-April 2, 2020. MMWR Morbidity and Mortality Weekly Report 6 April 2020 [cited 2020 May 26]. Available from: doi: 10.15585/mmwr.mm6914e4.

[5] Shekerdemian LS, Mahmood NR, Wolfe KK, Riggs BJ, Ross CE, McKiernan CA, et al. Characteristics and Outcomes of Children With Coronavirus Disease 2019 (COVID-19) Infection Admitted to US and Canadian Pediatric Intensive Care Units. JAMA Pediatr. 2020.

[6] Young NL, Gilbert TK, McCormick A, Ayling-Campos A, Boydell K, Law M, et al. Youth and young adults with cerebral palsy: their use of physician and hospital services. Archives of Physical Medicine and Rehabilitation. 2007; 88(6): 696-702.

[7] Naidu K, Smith K, Sheedy M, Adair B, Yu X, Graham HK. Systemic adverse events following botulinum toxin A therapy in children with cerebral palsy. Developmental Medicine and Child Neurology. 2010; 52(2): 139-44.

[8] Montastruc J, Marque P, Moulis F, Bourg V, Lambert V, Durrieu G, et al. Adverse drug reactions of botulinum neurotoxin type A in children with cerebral palsy: a pharmacoepidemiological study in VigiBase. Developmental Medicine and Child Neurology. 2017; 59(3): 329-34.
[9] Swinney CM, Bau K, Burton KLO, O’Flaherty SJ, Bear NL, Paget SP. Severity of cerebral palsy and likelihood of adverse events after botulinum toxin A injections. Developmental Medicine and Child Neurology. 2018; 60(5): 498-504.

[10] Paget SP, Swinney CM, Burton KLO, Bau K, O'Flaherty SJ. Systemic adverse events after botulinum neurotoxin A injections in children with cerebral palsy. Developmental Medicine and Child Neurology. 2018; 60(11): 1172-7.

[11] Calis EA, Veugelers R, Sheppard JJ, Tibboel D, Evenhuis HM, Penning C. Dysphagia in children with severe generalized cerebral palsy and intellectual disability. Developmental Medicine and Child Neurology. 2008; 50(8): 625-30.

[12] Control CfD. Interim infection prevention and control recommendations for patients with suspected or confirmed coronavirus disease 2019 (COVID-19) in healthcare settings [cited 2020 May 26]. Available from: https://www.cdc.gov/coronav irus/2019-ncov/hcp/infection-control-recommendations.html.

[13] Paulson A, Zigler CK, Houtrow A, Pruitt D. Botulinum toxin: techniques within pediatric physiatry. PM \& R: The Journal of Injury, Function, and Rehabilitation. 2019; 11(1): 38-44.

[14] Kishore M, Schaeffer E, Sanders J, Zaltz I, Kocher M. Evidence-based recommendations for pediatric orthopaedic practice. Journal of Pediatric Orthopedics. 2018 Oct; 38(9): e551-e555.

[15] Hastings-Ison T, Blackburn C, Rawicki B, Fahey M, Simpson $\mathrm{P}$, Baker R, Graham K. Injection frequency of botulinum toxin a for spastic equinus: a randomized clinical trial. Developmental Medicine and Child Neurology. 2016; 58: 753-757.

[16] Muktani I, Manji K, Hasting-Ison T, Khot A, Graham, K. Botulinum toxin management and children with cerebral palsy. Pediatric Drugs. 2019; 21: 260-281.13. 